ISSN 1870-4069

\title{
CAD design and Identification of Hydrodynamic Parameters of an Underwater Glider Vehicle
}

\author{
Jorge Díaz Moreno ${ }^{1}$, Reynaldo Ortíz Pérez ${ }^{1}$, Eduardo Campos Mercado ${ }^{1}$, \\ Luis Fidel Cerecero Natale ${ }^{1,2}$ \\ ${ }^{1}$ Universidad Politécnica de Pachuca, Posgrado en Mecatrónica, \\ Zempoala, Hgo., Mexico \\ ${ }^{2}$ Instituto Tecnológico de Cancún, Ingeniería en Mecatrónica, \\ Cancún, Quintana Roo, Mexico \\ jorge.diaz@micorreo.upp.edu.mx
}

\begin{abstract}
This article describes the design, modeling and identification of the main hydrodynamic parameters of an underwater glider vehicle is presented. The equations describing the dynamics of the vehicle is obtained from the Euler-Lagrange method. The main hydrodynamic parameters were obtained considering the geometry of the vehicle and its operating characteristics. Finally, simulation open loop system are presented.
\end{abstract}

Keywords: Hydrodynamic parameters, underwater glider vehicle.

\section{Introduction}

Unmanned underwater vehicles are classified in ROV's (Remotely Operated Vehicle) and AUV vehicles (Autonomous Underwater Vehicles) [8]. The Gliders are a type of AUV that control their own buoyancy and can spend long periods of time under water due to its autonomy low power consumption, because they usually do not use propellers or cables to the ship [4]. The diversity of underwater activities is wide and Gliders have efficient ways to perform tasks such as: to contribute to research, inspections and assessments, exploration of marine flora and fauna, deep-sea exploration for oil projects, search and rescue of objects in areas risk to humans [7].

The Gliders are underwater vehicles designed to carry out exploration, inspection, maintenance and surveillance, among others [8]. Most gliders have a cylindrical shape, this to minimize the effect of hydrodynamic damping and consequently the lower the energy required for vehicle propulsion [6].

The same laws of hydrodynamics governing the flow of the medium and the forces for air carriers can apply for underwater vehicles, but due to the high density of water (800 times greater than air) add effects buoyancy and masses added that they are significant in the dynamics of Glider [9]. The characteristic movement of a Glider describes a pattern in the form of sawtooth as shown in 


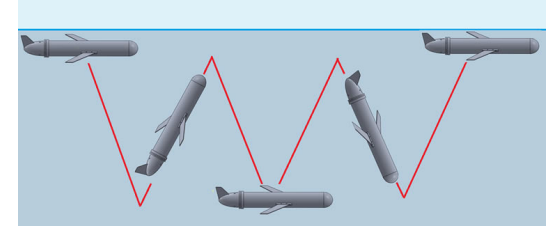

Fig. 1: Characteristic movement of a Glider.

Figure 1. They can carry sensors to measure physical environmental parameters, biogeochemical, acoustic and collect data on chlorophyll, I oxygen temperature, salinity [1] and if so, to estimate undercurrents or maritime density gradients, reproduction and primary study marine ecosystems, research on tracking whales and other marine mammals, among others [13].

Although usually these vehicles travel at low speed (average $0.4 \mathrm{~m} / \mathrm{s}[9]$ ), Glider design was adapted to improve performance in displacement and hydrodynamic forces, with the help of several sketches based on the mechanical design methodology Nigel Cross [10].

\section{Description of a Glider}

Gliders geometry is important for propulsion and maneuverability factor at different speeds. One of the most common ways in Gliders is torpedo-shaped consisting of a cylindrical body with its conical or spherical tips to simplify identification of the hydrodynamic parameters [11]. Accordingly, the geometry of the vehicle UPPGlider presented in this paper it is cylindrical, with a diameter of $15.4 \mathrm{~cm}$ and a length of $90 \mathrm{~cm}$, see Figure 2. Based on the design of the vehicle and in order to simplify the mathematical model of UPPGlider, Assuming that the vehicle is moving at low speed, that is $0.25 \mathrm{~m} / \mathrm{s}$.

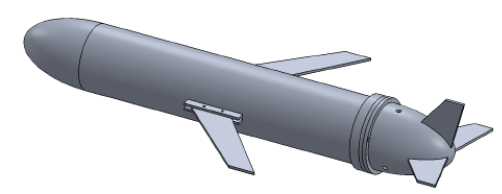

Fig. 2: UPPGlider: CAD design of the underwater vehicle.

Immersion and emersion (offset from the axis $Z_{I}$ ) vehicle is generated by changing its buoyancy ballast tank, while the displacement in the plane $X_{I}, Y_{I}$ is produced by the wings, the orientation of the vehicle and changing buoyancy [3]. The angular position roll $(\phi)$ remains close to zero due to the location of the 
center of buoyancy and the center of gravity of the vehicle, the side wings help the damping roll; while the rotational movement pitch $(\theta)$ It is produced by the effect of a moving mass and rotation yaw $(\psi)$ It is governed by a rudder that is located in the tail of the vehicle [2].

The embedded system consists of a computer $2.9 \mathrm{GHz}$ Intel Core i7-3520M, 8GB of RAM, Windows 8.1 operating system and Visual Studio C ++ 2010 to program the control strategy. Measurements of the inertial unit and the pressure sensor are performed with a USB-I2C interface connected directly to the PC. The main characteristics of the vehicle are described in Table 1.

Table 1: Main features of the UUP Glider.

\begin{tabular}{ll}
\hline \hline Dough & $15 \mathrm{~kg}$ \\
Buoyancy & $9 \mathrm{~N}$ \\
Dimensions & $90 \mathrm{~cm}(\mathrm{l}) \times 15.4 \mathrm{~cm}(\mathrm{w}) \times 15.4 \mathrm{~cm}(\mathrm{~h})$ \\
Operating depth & $100 \mathrm{~m}$ \\
Operating Voltage & $14 \mathrm{~V}$ \\
Computer & NUC intel core i $-2.9 \mathrm{GHz}$ \\
& Windows 8.1 Professional $64 \mathrm{bits}$ \\
& Microsoft Visual C++ 2010 \\
\hline
\end{tabular}

\section{Mathematical Model}

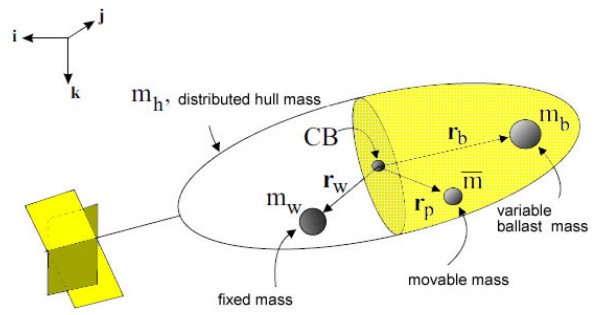

Fig. 3: Definition of mass of the Glider. [5]

The vehicle is modeled as a rigid body immersed in a fluid with fixed wings and tail, with the particularity that the total vehicle mass $m_{v}$, is the sum of the mass of the hull $m_{h}$, calibration mass $m_{w}$, the moving mass $\bar{m}$ and mass ballast tank $m_{b}$; whose location with respect to the coordinate system fixed to the vehicle body, is described by the vectors $r_{h}=0, r_{w}, r_{p}$ y $r_{b}$ respectively, as shown in 
Figure 3. It is noteworthy that the mass ballast tank is variable $\left(m_{b}\right)$ but their position is constant, whereas the moving mass $(\bar{m})$ It is constant but is variable position.

To describe the force produced by the buoyancy has to the mass of the fluid displaced by the vehicle is denoted by $m$. Consequently, the net buoyancy is defined as: $m_{0}=m_{v}-m$ so the vehicle has positive buoyancy if $m_{0}>0$.

The equations describing the dynamics of Glider are obtained starting in the momentum, which you have to $p$ It represents the moment of translation of the vehicle and the fluid and $\pi$ the angular momentum, momentum both expressed respect to the inertial coordinate system. In the case of translational momentum is must $p_{p}$ It represents the total momentum of the moving mass $\bar{m}, p_{b}$ they represent the momentum of the mass of ballast tank $m_{b}$ and $p_{w}$ describes the momentum of the mass $m_{w}$. Considering the above and Newton's laws we have:

$$
\begin{aligned}
& \dot{p}=\sum_{i=1}^{I} f_{\text {ext }}, \\
& \dot{\pi}=\sum_{i=1}^{I}\left(x_{i} \times f_{\text {ext }_{i}}\right)+\sum_{j=1}^{J} \tau_{\text {ext }_{j}}, \\
& \dot{p}_{p}=\bar{m} g \boldsymbol{k}+\sum_{k=1}^{K} f_{\text {int_pointmass }_{k}}, \\
& \dot{p}_{b}=m_{b} g \boldsymbol{k}+\sum_{l=1}^{L} f_{\text {int_ballast }_{l}}, \\
& \dot{p}_{w}=m_{w} g \boldsymbol{k}+\sum_{n=1}^{N} f_{\text {int_w }_{n}},
\end{aligned}
$$

where $k$ is the unit vector in the direction of gravity, $f_{e x t_{i}}$ an external force is applied to the system and $\tau_{\text {ext }}$ It is an external torque. The vector $x_{i}$ The vector is the location of the application point of force $f_{\text {ext }}$ with respect to the inertial coordinate system. These external forces and torques include those due to gravity and buoyancy; however, gravity is explicitly included in the last three equations because it is the only force acting on the three masses. The force $f_{\text {int_pointmass }}$ is applied from the vehicle body to the mobile hub, it will be used as a control input. The forces $f_{\text {int_ballast }_{l}}$ y $f_{w_{n}}$ are the forces applied from the vehicle body to the ballast system and mass calibration respectively.

In order to obtain the representation of the mathematical model with respect to the coordinate system fixed in the vehicle, it has to $P$ It represents the momentum of the vehicle and $\Pi$ the angular momentum, both expressed in the coordinate system of the vehicle. The momentum of the moving mass is represented by $P_{p}$, on the other hand; $P_{b}$ y $P_{w}$ They represent the momentum of the mass of the ballast tank and the mass calibration of the vehicle respectively. From the above it has the following expression:

$$
\begin{aligned}
& p=R P, \\
& \pi=R \Pi+b \times p, \\
& p_{p}=R P_{p}, \\
& p_{b}=R P_{b}, \\
& p_{w}=R P_{w} .
\end{aligned}
$$


Deriving the equations in (2) and using the expressions of kinematics and by substituting equation (1) it follows that:

$$
\begin{gathered}
\dot{P}=P \times \Omega+R^{T} \sum_{i=1}^{I} f_{\text {ext }}, \\
\dot{\Pi}=\Pi \times \Omega+P \times \nu+R^{T}\left(\sum_{i=1}^{I}\left(x_{i}-b\right) \times f_{\text {ext }}\right)+R^{T} \sum_{j=1}^{J} \tau_{\text {ext }}, \\
\dot{P}_{p}=P_{p} \times \Omega+\bar{m} g\left(R^{T} k\right)+R^{T} \sum_{k=1}^{K} f_{\text {int_pointmass }_{k}} \\
\dot{P}_{b}=P_{b} \times \Omega+m_{b} g\left(R^{T} k\right)+R^{T} \sum_{l=1}^{L} f_{\text {int_ballast }_{l}} \\
\dot{P}_{w}=P_{w} \times \Omega+m_{w} g\left(R^{T} k\right)+R^{T} \sum_{n=1}^{N} f_{\text {int_w }_{n}},
\end{gathered}
$$

where $R^{T} \sum_{k=1}^{K} f_{\text {int_pointmass }_{k}}$ is the internal force acting on the mass point $\bar{m}$ in the coordinates of the vehicle body. Be:

$$
\bar{u}=\left[\begin{array}{l}
\bar{u}_{1} \\
\bar{u}_{2} \\
\bar{u}_{3}
\end{array}\right]=P_{p} \times \Omega+\bar{m} g\left(R^{T} k\right)+R^{T} \sum_{k=1}^{K} f_{\text {int_pointmass }_{k}} .
$$

such that

$$
\dot{P}_{p}=\bar{u}
$$

$\bar{u}$ internal strength is not the point mass in gravitational forces because centrifuged $\bar{m}$ they have been included in the expression for $\bar{u}$, also is:

$$
\begin{gathered}
u_{b}=\left[\begin{array}{l}
u_{b 1} \\
u_{b 2} \\
u_{b 3}
\end{array}\right]=P_{b} \times \Omega+m_{b} g\left(R^{T} k\right)+R^{T} \sum_{l=1}^{L} f_{\text {int_ballast }_{l}}, \\
u_{w}=\left[\begin{array}{l}
u_{w 1} \\
u_{w 2} \\
u_{w 3}
\end{array}\right]=P_{w} \times \Omega+m_{w} g\left(R^{T} k\right)+R^{T} \sum_{n=1}^{N} f_{\text {int_w }} .
\end{gathered}
$$

So:

$$
\begin{gathered}
\dot{P}_{b}=u_{b}, \\
\dot{P}_{w}=u_{w} .
\end{gathered}
$$

The terms $\frac{d}{d t} I^{-1}(i, j)$ They are usually small in existing Glider. $\dot{r}_{w} \mathrm{y} \dot{r}_{b}$ they are zero. $\dot{r}_{p}$ it is zero in balancing the gliders and is small during transitions 
between equilibrium (due to the speed of actuators Glider). The magnitudes of the elements $J$ generally they are large compared with the elements of $r_{p}, r_{b}$ y $r_{w}$. The terms may be significant in designs Gliders very large or very small actuators or those with fast internal mass.

The full equations of motion for a submarine Glider in three dimensional space are:

$$
\left[\begin{array}{c}
\dot{R} \\
\dot{b} \\
\dot{\Omega} \\
\dot{v} \\
\dot{r}_{p} \\
\dot{r}_{b} \\
\dot{r}_{w} \\
\dot{P}_{p} \\
\dot{P}_{b} \\
\dot{P}_{w} \\
\dot{m}_{b}
\end{array}\right]=\left[\begin{array}{c}
R \hat{\Omega} \\
R v \\
J^{-1} \bar{T} \\
M^{-1} \bar{F} \\
\frac{1}{\bar{m}} P_{p}-v-\Omega \times r_{p} \\
\frac{1}{m_{b}} P_{b}-v-\Omega \times r_{b} \\
\frac{1}{m_{w}} P_{w}-v-\Omega \times r_{w} \\
\bar{u} \\
u_{b} \\
u_{w} \\
u_{\text {ballast_rate }}
\end{array}\right]
$$

where:

$$
\begin{aligned}
\bar{T}= & \left(J \Omega+\hat{r}_{p} P_{p}+\hat{r}_{b} P_{b}+\hat{r}_{w} P_{w}\right) \times \Omega+(M v \times v) \\
& +\left(\Omega \times r_{p}\right) \times P_{p}+\left(\Omega \times r_{b}\right) \times P_{b}+\left(\Omega \times r_{w}\right) \times P_{w}+\left(\bar{m} \hat{r}_{p}+m_{b} \hat{r}_{b}+m_{w} \hat{r}_{w}\right) g R^{T} k \\
& +T_{e x t}-\hat{r}_{p} \bar{u}-\left(\hat{r}_{b} u_{b}+\hat{r}_{w} u_{w}\right), \\
\bar{F}= & \left(M v+P_{p}+P_{b}+P_{w}\right) \times \Omega+m_{0} g R^{T} k+F_{e x t}-\bar{u}-\left(u_{b}+u_{w}\right) .
\end{aligned}
$$

Thus,

$$
\begin{aligned}
& F_{e x t}=R^{T} \sum f_{e x t_{i}}, \\
& T_{e x t}=R^{T} \sum\left(x_{i}-b\right) \times f_{\text {ext }}+R^{T} \sum \tau_{\text {ext }},
\end{aligned}
$$

where $x_{i}$ is the point, where the inertial system $f_{\text {ext }}$ acts represents external forces and moments including lift, drag and hydrodynamic moments associated with the system of the vehicle.

$\bar{u}$ is a force control $\bar{m}$, and $u_{b}$ and $u_{w}$ are internal forces in the masses $m_{b}$ and $m_{w}$. They will be restoring forces when the masses are fixed in one place.

\section{Hydrodynamic Parameters Identification}

When a body immersed in a fluid moves; a quantity of fluid is moved along with it (added mass), this amount of fluid will depend on the geometry of the vehicle [5], Likewise; There forces affecting movement direction in $x, y, z$ (Hydrodynamic forces) and moments affecting the orientation roll $(\phi)$, pitch $(\theta)$, and yaw $(\psi)$ (hydrodynamic moments) [4].All measurements are made based on the center of buoyancy, because at this point systems coordinates fixed to the vehicle and the reference coordinate system are located fluid. 


\subsection{Aggregated mass}

The added mass is a function of an increase in mass and thus increased acceleration inercia.Esas forms are represented by the added mass $(M f)$ added inertia $(J f)$ and cross terms $(D f)$ :

$$
M_{A}=\left[\begin{array}{cc}
M_{f} & D_{f}^{T} \\
D_{f} & J_{f}
\end{array}\right]
$$

Approximating the diagonal is considered acceptable, since the elements outside the diagonal are much smaller than diagonal elements itself, this is because the vehicle moves at low speed, so that the mass matrix added $M_{A}$ is defined as:

$$
M_{A}=-\left[\begin{array}{cccccc}
X_{\dot{u}} & 0 & 0 & 0 & 0 & 0 \\
0 & Y_{\dot{v}} & 0 & 0 & 0 & 0 \\
0 & 0 & Z_{\dot{w}} & 0 & 0 & 0 \\
0 & 0 & 0 & K_{\dot{p}} & 0 & 0 \\
0 & 0 & 0 & 0 & M_{\dot{q}} & 0 \\
0 & 0 & 0 & 0 & 0 & N_{\dot{r}}
\end{array}\right]
$$

where $X_{\dot{u}}, Y_{\dot{v}}$ and $Z_{\dot{w}}$ is the added mass on the axle $x, y$ and $z$ respectively. $K_{\dot{p}}, M_{\dot{q}}$ and $N_{\dot{r}}$ are the moments of inertia about the axis added $x, y$ and $z$ respectively.

The equations describing each element of the diagonal matrix $M_{A}$ based on the geometry proposed Glider, they are obtained from the vehicle profile Figure 4 ; the values are presented in table 2 .

Table 2: Parameters of Figure 4.

\begin{tabular}{|c|c|c|l|}
\hline Parameter & Value & Units & Description \\
\hline$D_{b_{1}}$ & 168.28 & $\mathrm{~mm}$ & Diameter section $b_{1}$ \\
\hline$D_{b_{2}}$ & 190.5 & $\mathrm{~mm}$ & Diameter section $b_{2}$ \\
\hline$a$ & 84.14 & $\mathrm{~mm}$ & Length of the nose \\
\hline$b_{1}$ & 750 & $\mathrm{~mm}$ & Section length $b_{1}$ \\
\hline$b_{2}$ & 43 & $\mathrm{~mm}$ & Section length $b_{2}$ \\
\hline$b$ & 793 & $\mathrm{~mm}$ & Total length of the cylindrical section \\
\hline$c$ & 190 & $\mathrm{~mm}$ & Queue Length \\
\hline
\end{tabular}

In our case, we assume that the profile of the vehicle starts at the source positioning system coordinates in the nose of the vehicle, both the nose and tail are symmetrical, so they are governed by the equation of the ellipse on the horizontal axis outside the origin: 


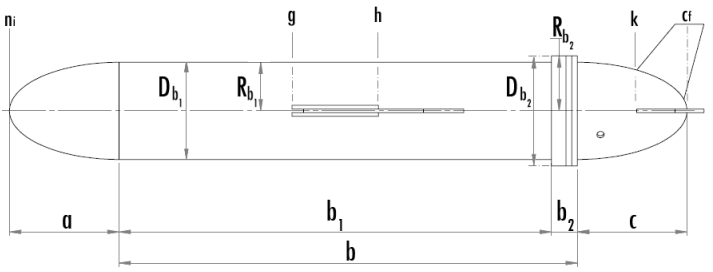

Fig. 4: Proposed Vehicle Profile.

$$
\frac{\left(x-h_{e}\right)^{2}}{a_{e}^{2}}+\frac{\left(y-k_{e}\right)^{2}}{b_{e}^{2}}=1 .
$$

Whereas the only center axis moves $x$ so $k_{e}=0$ and $y$, as function has:

$$
y=\sqrt{\left(1-\frac{\left(x-h_{e}\right)^{2}}{a_{e}^{2}}\right) b_{e}^{2}} .
$$

The function $y$ It is equal to the radius of the nose or tail of the hull so it can be considered for both cases $y=R(x)$ and Sections $b_{1}$ and $b_{2}$ Radio function $R(x)$ It can be considered constant.

These equations define the profile of the nose and tail of the vehicle without considering the fins, stabilizers, rudders or control surfaces.

Axial Added Mass $\left(\boldsymbol{X}_{\dot{\boldsymbol{u}}}\right)$ To determine the axial added mass is considered the variation of vehicle shape in areas of the bow and stern:

$$
\begin{gathered}
X_{\dot{u}}=-m_{11}=-\frac{4 \alpha_{f} \rho \pi}{3}\left(\frac{l}{2}\right)\left(\frac{D_{b_{1}}}{2}\right)^{2}, \\
X_{\dot{u}}=-m_{11}=-\frac{4 \beta_{f} \rho \pi}{3}\left(\frac{d_{b_{1}}}{2}\right)^{3},
\end{gathered}
$$

where $\rho$ To determine the axial added mass is considered the variation of vehicle shape in areas of the bow and stern. $\rho=1030 \frac{\mathrm{kg}}{\mathrm{m}^{3}}, \alpha_{f}$ y $\beta_{f}$ are parameters determined by the ratio of the length to the diameter of the vehicle according to Tables Blevins [14], such that on average; $\alpha_{f}=0.047485$ y $\beta_{f}=0.2733$ this because the value of $\frac{l}{D_{b_{1}}}$ y $\frac{l}{D_{b_{2}}}$ values are respectively 5 to 7 .

Added Mass Crossflow $\left(\boldsymbol{Y}_{\dot{\boldsymbol{v}}}, \boldsymbol{Z}_{\dot{\boldsymbol{w}}}, \boldsymbol{M}_{\dot{q}}, \boldsymbol{N}_{\dot{\boldsymbol{r}}}\right)$ The added mass is calculated by the Strip Theory [12] It applied to the cross sections of the hull and consists in dividing the submerged part of the vehicle in a series of slices to determine the hydrodynamic coefficients of each section in two dimensions and be integrated 
along the length of the body finally obtain the coefficients in three dimensions. Aggregate per unit length of a single cylindrical mass segment is given by:

$$
m_{a}(x)=\pi \rho R(x)^{2},
$$

where $\rho$ It is the density of the surrounding fluid and $R(x)$ is the radius of the hull as a function of axial position. The aggregate mass finned a circle is given by:

$$
m_{a f}=\pi \rho\left(a_{\text {aleta }}^{2}-R(x)^{2}+\frac{R(x)^{4}}{a_{\text {aleta }}^{2}}\right),
$$

where $a_{\text {aleta }}$ It is the maximum height of the fin measured from the axis of the ellipsoid. By integrating the equations 17 and 18 the length of the vehicle is reached:

$$
\begin{aligned}
& Y_{\dot{v}}=-\int_{n_{i}}^{g} m_{a}(x) d x-\int_{g}^{h} m_{a f}(x) d x- \\
& -\int_{h}^{k} m_{a}(x) d x-\int_{k}^{c_{f}} m_{a f}(x) d x, \\
& Z_{\dot{w}}=-\int_{n_{i}}^{c_{f}} m_{a}(x) d x, \\
& M_{\dot{q}}=-\int_{n_{i}}^{g} x^{2} m_{a}(x) d x-\int_{g}^{h} x^{2} m_{a f}(x) d x- \\
& -\int_{h}^{k} x^{2} m_{a}(x) d x-\int_{k}^{c_{f}} x^{2} m_{a f}(x) d x, \\
& N_{\dot{r}}=-\int_{n_{i}}^{c_{f}} x^{2} m_{a}(x) d x .
\end{aligned}
$$

Due to Added Mass Balance $\left(\boldsymbol{K}_{\dot{p}}\right)$ To estimate the added mass due to rolling, only it considered the section of the hull containing the fins [12]:

$$
K_{\dot{p}}=-\int_{g}^{h} \frac{2}{\pi} \rho a_{\text {aleta }}^{4} d x .
$$

Substituting measures and functions, parameters are obtained aggregate mass $M_{A}$.

Table 3: Results of the added mass.

\begin{tabular}{|c|c|c|}
\hline Parameter & Value & Units \\
\hline$X_{\dot{u}}$ & -0.7738890384 & $\mathrm{~kg}$ \\
\hline$Y_{\dot{v}}$ & -60.49746284 & $\mathrm{~kg}$ \\
\hline$Z_{\dot{w}}$ & -22.49920646 & $\mathrm{~kg}$ \\
\hline$K_{\dot{p}}$ & -0.8306898369 & $\frac{\mathrm{kg} \cdot \mathrm{m}^{2}}{\mathrm{rad}}$ \\
\hline$M_{\dot{q}}$ & -15.85575167 & $\frac{\mathrm{kg} \cdot \mathrm{m}^{2}}{\mathrm{rad}}$ \\
\hline$N_{\dot{r}}$ & -7.843744090 & $\frac{\mathrm{kg} \cdot \mathrm{m}^{2}}{\mathrm{rad}}$ \\
\hline
\end{tabular}

\subsection{Aerodynamic Forces and Moments}

The forces $F_{\text {hydro }}$ and aerodynamic moments $M_{\text {hydro }}$ viscous effects appear water during movement of the glider and are usually expressed under the fluid as [14]: 


$$
\begin{gathered}
F_{\text {hydro }}=\left[\begin{array}{c}
-D \\
S F \\
-L
\end{array}\right]=\left[\begin{array}{l}
\left(K_{D 0}+K_{D} \alpha^{2}\right) V^{2} \\
K_{\beta} \beta V^{2} \\
\left(K_{L 0}+K_{\alpha} \alpha\right) V^{2}
\end{array}\right], \\
M_{\text {hydro }}=\left[\begin{array}{l}
T_{D L 1} \\
T_{D L 2} \\
T_{D L 3}
\end{array}\right]=\left[\begin{array}{l}
\left(K_{M R} \beta+K_{p} p\right) V^{2} \\
\left(K_{M 0}+K_{M} \alpha+K_{q} q\right) V^{2} \\
\left(K_{M Y} \beta+K_{r} r\right) V^{2}
\end{array}\right],
\end{gathered}
$$

where: $K_{D 0}, K_{D}$ are coefficients of the drag force $(x)$ in $D$ with respect to $V^{2}$, $\alpha^{2} V^{2}$.

$K_{\beta}$ It is the coefficient of force $(y)$ side $L$ with respect to $\beta V^{2}$.

$K_{L 0}, K_{\alpha}$ are coefficients of force $(z)$ supporting $L$ with respect to $V^{2}, \alpha V^{2}$.

$K_{M R}, K_{p}$ are coefficients roll moment $T_{D L 1}$ with respect to $\beta V^{2}, p V^{2}$.

$K_{M 0}, K_{M}, K_{q}$ are coefficients pitch moment $T_{D L 2}$ with respect to $V^{2}, \alpha V^{2}$, $q V^{2}$.

$K_{M Y}, K_{r}$ coefficients are time yaw $T_{D L 3}$ with respect to $\beta V^{2}, r V^{2}$.

Analysis of the Data To determine the values of the coefficients of the hydrodynamic forces and moments has been used data collection through various fluid simulations with CFD module called SolidWorks Flow Simulation. The simulations are to be changing angles of attack $\alpha$ and sideslip $\beta$, where:

$$
\begin{gathered}
\alpha=\tan ^{-1}\left(\frac{V_{3}}{V_{1}}\right), \\
\beta=\tan ^{-1}\left(\frac{V_{2}}{\sqrt{V_{1}^{2}+V_{2}^{2}+V_{3}^{2}}}\right) .
\end{gathered}
$$

The coordinate system flow shown in Figure 5 together with the angles of attack $\alpha$ and sideslip $\beta$ plus speeds on each axis.
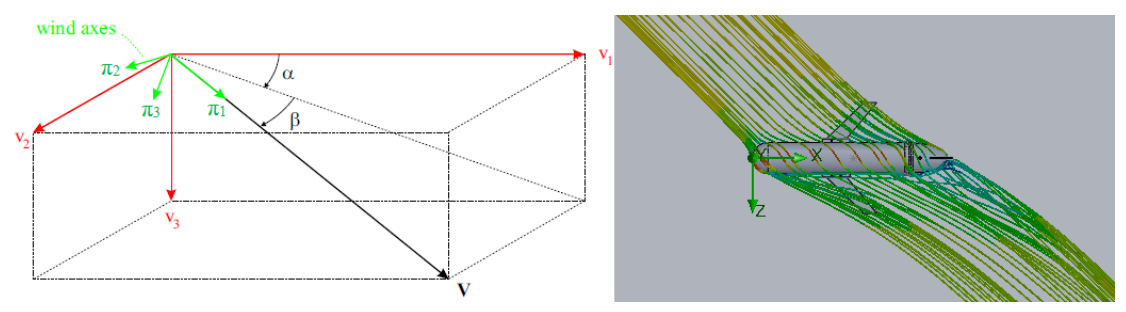

Fig. 5: Vehicle reference systems and fluid and Fluid direction in Flow Simulation respectively. 
Glider speed is represented transforming the speed displayed in the fluid system attached to the body system as:

$$
\left[\begin{array}{l}
V_{1} \\
V_{2} \\
V_{3}
\end{array}\right]=R_{W B} \times\left[\begin{array}{l}
V \\
0 \\
0
\end{array}\right]=\left[\begin{array}{c}
V \cos \alpha \cos \beta \\
V \sin \beta \\
V \cos \beta \sin \alpha
\end{array}\right] .
$$

To determine the values of the coefficients of the hydrodynamic forces in these simulations is configured that the total speed that will move the glider is $V=\sqrt{V_{1}^{2}+V_{2}^{2}+V_{3}^{2}}=0.5 \mathrm{~m} / \mathrm{s}$, varying angles $\alpha$ and $\beta$ It is to vary the velocity components of the fluid and then let the software determine the hydrodynamic forces.

The table 4 shows the values of the obtained coefficients.

Table 4: Values of the constants of aerodynamic forces and moments.

\begin{tabular}{|c|c|c|}
\hline Parameter & Value & Units \\
\hline$K_{D 0}$ & 5.04 & $\mathrm{~kg} / \mathrm{m}$ \\
\hline$K_{D}$ & -4.2863 & $\mathrm{~kg} / \mathrm{m} / \mathrm{rad}^{2}$ \\
\hline$K_{\beta}$ & -42.3403 & $\mathrm{~kg} / \mathrm{m} / \mathrm{rad}$ \\
\hline$K_{L 0}$ & 0.7 & $\mathrm{~kg} / \mathrm{m}$ \\
\hline$K_{\alpha}$ & 95.3556 & $\mathrm{~kg} / \mathrm{m} / \mathrm{rad}$ \\
\hline$K_{M R}$ & -16.7381 & $\mathrm{~kg} / \mathrm{rad}$ \\
\hline$K_{p}$ & -174.3244 & $\mathrm{~kg} \mathrm{~s} / \mathrm{rad}$ \\
\hline$K_{M 0}$ & 0.392 & $\mathrm{~kg}$ \\
\hline$K_{M}$ & -40.2293 & $\mathrm{~kg} / \mathrm{rad}$ \\
\hline$K_{q}$ & -117.804 & $\mathrm{~kg} \mathrm{~s} / \mathrm{rad}$ \\
\hline$K_{M Y}$ & 20.794 & $\mathrm{~kg} / \mathrm{rad}$ \\
\hline$K_{r}$ & -41.2834 & $\mathrm{~kg} \mathrm{~s} / \mathrm{rad}$ \\
\hline
\end{tabular}

\section{$5 \quad$ Simulation and Results}

In Figure 6 the mathematical model in MatLab Simulink shown. Table data used 3 column of the results according to the equations describing the actual profile and the values of constants Table 4 .

The conditions for the simulation were that the vehicle has a rudder that between 50 and 210 seconds is kept rotated 20 degrees and 210 to 410 seconds is maintained at -20 degrees while the rest of the time is kept 0 . moving mass changes $r_{p}=[0.3,0,0]$ to $r_{p}=[-0.3,0,0]$ and the value of $m_{b}$ changes from 0.5 to -0.5 every 30 seconds initial conditions are: $x=0, y=0, z=0, \phi=0, \theta=0$, $\psi=0$ and $\nu=0$. The result can be seen in the graphs of Figures 6,7 and 8 .

You can see that the roll angle $(\phi)$, practically remains unchanged due to the fixed side wings in the vehicle, the yaw angle $(\psi)$ obeys the programmed rotation of the wheel, while the angle pitch $(\theta)$ semiperidico shows the constant pitch all the way due to the change of the moving mass $r_{p}$ and $m_{b}$ every 30 seconds. 

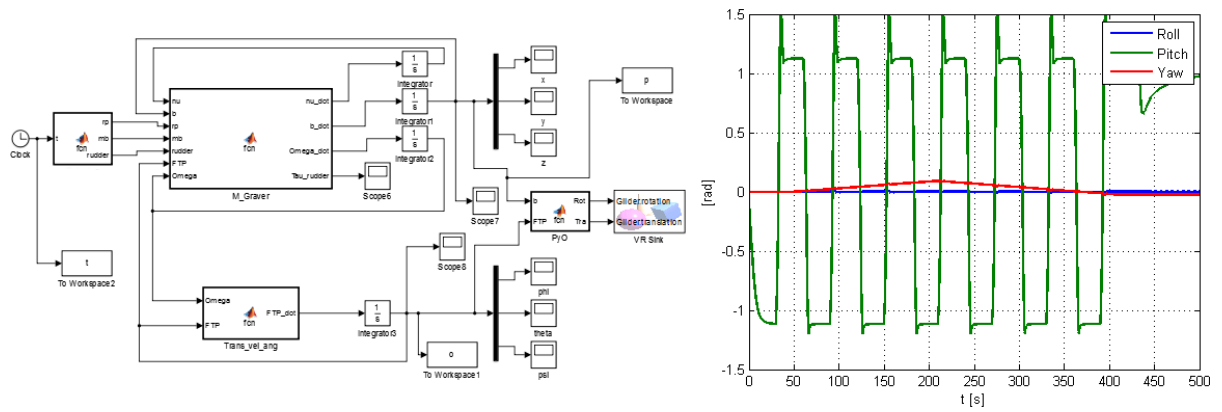

Fig. 6: Graver model in Simulink and Orientation respectively.

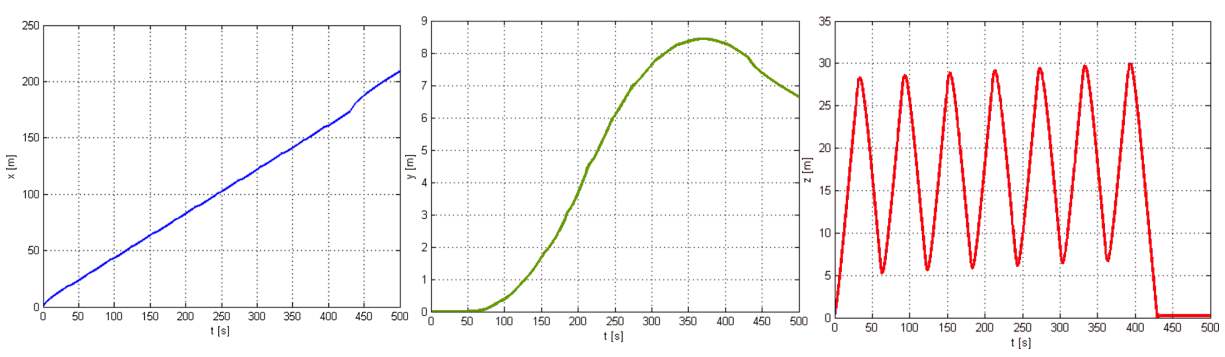

Fig. 7: Displacement in $\mathrm{X}, \mathrm{Y}$, and Z respectively.

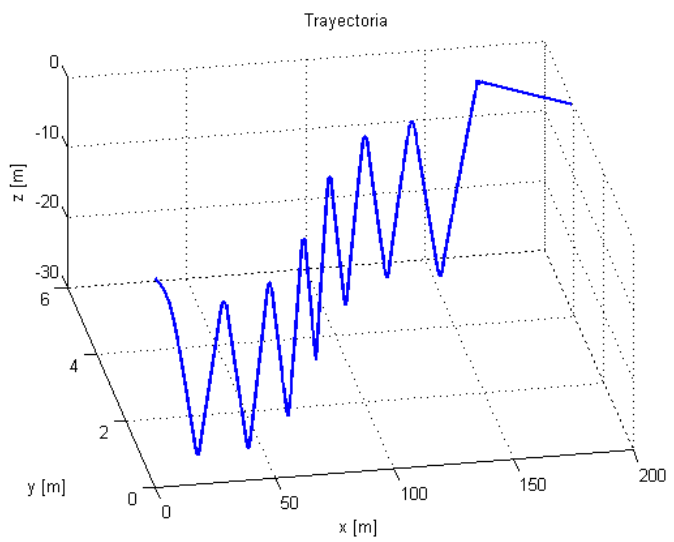

Fig. 8: Position.

Also, in the printing position, it can be seen that the vehicle travels a slightly longer distance on the shaft $250 \mathrm{~m} X$ during the duration of the test 500 s travel, while displacement in $Y$ the way evidence produced due to scheduling helm of the vehicle and finally the characteristic zig-zagueo the Glider due to the angle in pitch $(\theta)$ and the wings of the vehicle. 
Acknowledgments. This work was partially supported by a CONACyT CVU: 637204.

\section{References}

1. Akyildiz, I.F., Pompili, D., Melodia, R.: Underwater acoustic sensor networks: research challenges. Science Direct (2005)

2. Bender, A., Matthew, D., Lee, A., Williams, S.B.: Analysis of an autonomous underwater glider. Australian Centre for Field Robotics (2006)

3. Campos, E., J.Torres, S.Mondi, Lozano, R.: Deep control using artificial vision with time-delay of an auv. 9th International Conference on Electrical Engineering, Computing Science and Automatic Control (2012)

4. Fossen, T.I.: Marine control systems guidance, navigation, and control of ships, rigs and underwater vehicles (2002)

5. Graver, J.G.: Underwater gliders: Dynamics, control and design (2005)

6. Jun, B.H., Park, J.Y., Lee, F.Y., Lee, P.M., Lee, C.M., Kin, K., Lim, Y.K., Oh, J.H.: Development of the auv 'isimi' and a free running test in an ocean engieering basin. Ocean Engineering (2009)

7. Liang, X., Zhang, J., Qin, Y., Yang, H.: Dynamic modeling and onputer simulation for autonomous underwater vehicles with fins. Ocean Engineering (2012)

8. Mercado, E.C.: Design, modelling and control of auv's using artificial vision (2014)

9. Naomi Ehrich Leonard, J.G.G.: Model-based feedback control of autonomous underwater giders. IEE Journal of Oceanic Engineering, Vol. 26, No 4 (2001)

10. NigelCross: GEngeneering Design Methods Strategies for Product Design (2000)

11. Pellicer, A.G.: Estimacin de los coeficientes hidrodinmicos de vehculos autnomos submarionos mediante CFD

12. Prestero, T.: Verification of a six-degree of freedom simulation model for the remus autonomus underwater vehicle

13. Smith, S.N., Smith, S.L., Rus, D., ad Burton H. Jones, M.S., Sukhatme, G.S.: Persistent ocean monitoring with underwater gliders. Open Access Articles (2011)

14. Zhang, S., Yu, J., Zhang, A., Zhang, F.: Spilaring motion of underwater gliders: Modeling, analysis, and experimental results. Ocean Engineering (2012) 